\title{
Riconvertire gli edifici storici. II Complesso di San Michele a Ripa Grande a Roma e il Reale Albergo dei Poveri a Napoli ${ }^{1}$
}

\author{
Rosa Maria Giusto \\ Università degli Studi di Firenze \\ rosamaria.giusto@unifi.it
}

\begin{abstract}
RIASSUNTO: II contributo affronta il tema del riuso del patrimonio architettonico pubblico attraverso due esperienze eloquenti tra loro intimamente connesse riguardanti il Complesso monumentale di San Michele a Ripa Grande a Roma e il Reale Albergo dei Poveri a Napoli. Entrambe le strutture segnano, per mole e qualità del linguaggio adoperato, il tessuto urbano circostante costituendo esempi imponenti di quelle architetture dell'illuminismo pensate e progettate per assolvere a funzioni pubbliche e sociali. Le vicende evolutive del Complesso di San Michele a Ripa Grande presentano aspetti che possono indurci a guardare al caso romano come a un possibile modello di riferimento in tema di reusing di un edificio pubblico restituito nel tempo a una sua rinnovata funzione sociale e formativa, in grado di promuovere attivamente lo sviluppo del territorio urbano circostante.
\end{abstract}

PAROLE CHIAVE: San Michele a Ripa Grande; Reale Albergo dei Poveri; Riconversione; Rigenerazione urbana; Edifici storici.

\section{Conversion of Historic Buildings. The Complex of San Michele a Ripa Grande in Rome and the Reale Albergo dei Poveri in Naples}

ABSTRACT: The paper is focused on the theme of the reuse of public architectural heritage through two eloquent experiences, intimately connected to each other, concerning the monumental Complex of San Michele a Ripa Grande in Rome and the Reale Albergo dei Poveri in Naples. Both structures, for their dimensions and architectural language, mark the surrounding urban fabric, forming powerful examples of the architecture of Enlightenment designed to fulfill public and social functions. The transformation of the Complex of San Michele in Ripa Grande presents aspects that can lead us to consider the Roman case as a possible reference model in terms of reusing a public building, converted for a new social function, and able to promote the development of the surrounding urban area.

KEYWORDS: San Michele a Ripa Grande; Reale Albergo dei Poveri; Conversion; Urban Regeneration; Historic Buildings.

Recibido: 27 de febrero de 2018 / Aceptado: 16 de junio de 2018.

«La conservazione ha senso [...] non quando o solo quando si tratta di conservare la materia del monumento, ma quando e soprattutto quando si tratta di conservare il significato» (Marconi, 1999: 4).

«Gli edifici, come le persone, prendono certe abitudini delle quali è necessario tenere conto», dichiarava alla metà dell'Ottocento il padre fondatore del restauro stilistico Eugène Emmanuel Viollet Le Duc (1982: 247), ponendo l'accento sulla stretta relazione intercorrente tra la struttura degli edifici sottoposti ad attività di restauro e il loro temperamento, frutto di consuetudini e «vocazioni abitative» stratificatesi nel tempo.

Cómo citar este artículo: GIUSTO, Rosa Maria, «Riconvertire gli edifici storici. Il Complesso di San Michele a Ripa Grande a Roma e il Reale Albergo dei Poveri a Napoli», Boletín de Arte-UMA, n. ${ }^{\circ}$ 39, Departamento de Historia del Arte, Universidad de Málaga, 2018, pp. 113-124, ISSN: 0211-8483, DOI: http://dx.doi.org/10.24310/BoLArte.2018.v0i39.4221 
D'altra parte «restaurare un edificio non è conservarlo, ripararlo o rifarlo, è ripristinarlo in uno stato di completezza che può non essere mai esistito in un dato tempo» (Viollet Le Duc, 1982: 247): ciò vuol dire che il risultato finale, pur condotto secondo il temperamento e «l'anatomia» del manufatto, è il segno del proseguimento della sua storia evolutiva, di un suo sviluppo imprevisto, del protrarsi di una condizione che, intercettando tempi e bisogni diversi, è necessariamente altra da quella di partenza.

La difficoltà di rapportarsi con un patrimonio culturale vasto e diversificato qual è quello pubblico italiano pone questioni cogenti sul come intervenire nel pieno rispetto dei principi condivisi della conservazione integrata, allo stesso tempo prevedendone la piena fruibilità.

Il numero sempre crescente di edifici in disuso che il tempo e l'obsolescenza hanno consegnato al regesto delle opere impossibili da recuperare è tale d'aver indotto a tracciare vere e proprie geografie dell'abbandono dove sono attentamente elencate le numerose costruzioni dismesse, abbandonate o mai realmente impiegate, disseminate sul nostro territorio nazionale. In bilico tra memoria e obsolescenza, tali edifici domandano d'essere recuperati restituendo loro, almeno in parte, quella funzione ch'erano stati inizialmente chiamati a soddisfare.

Le metodologie d'intervento per la corretta re-immissione di tali complessi edilizi nel tessuto vitale delle città vanno nella direzione di restituire ad essi una rinnovata funzione, quanto più flessibile e omogenea in termini di tipologia e finalità, prevedendo soluzioni aperte a future modificazioni ed evoluzioni, unica strada percorribile per avviare politiche di salvaguardia attiva del patrimonio storico-artistico.

La necessità di prevedere interventi volti, da un lato, al recupero del bene sottoposto ad attività di salvaguardia, da un altro, al suo reimpiego funzionale, induce a guardare al problema del riuso da più punti di vista; le esperienze di rigenerazione possono rappresentare in tal senso «un'autentica leva di valorizzazione dei beni del patrimonio pubblico»² oltre che occasioni di sviluppo a vocazione culturale di interi brani di città degradati o rimasti ai margini delle politiche di riqualificazione dei singoli quartieri.

II recente moltiplicarsi di laboratori urbani e di bandi di idee e progetti finalizzati alla creazione di spazi per l'innovazione e l'inclusione sociale è indice della necessità, da più parti avvertita, di prevedere processi di sviluppo incentrati sulla riqualificazione di «spazi ibridi a vocazione culturale e creativa in cui [...] lavorare, produrre, distribuire, aggregare» (Tamiozzo, 2002).

Partendo dalla più moderna accezione di conservazione intesa non come difesa «passiva», ma come attività di tutela dinamica "comprensiva degli specifici profili della conoscenza, del restauro, del ripristino, della fruizione e della valorizzazione degli stessi beni» (Tamiozzo, 2002) e contro un'idea di tutela intesa come musealizzazione dell'architettura e della città, le vicende edilizie che hanno interessato il patrimonio storico-artistico nei secoli testimoniano i temi dell'innovazione funzionale e tecnologica, dell'ampliamento e della trasformazione delle tipologie storiche come conseguenza necessaria dell'adeguamento ai bisogni della società e come prima, «empirica», risposta ai temi della conservazione. Ma quale riorganizzazione delle strutture esistenti occorre immaginare in relazione alla loro dimensione, tipologia storica e collocazione sul territorio, anche in considerazione della sperimentazione di nuove tecniche e delle incalzanti esigenze di inclusività e superamento delle barriere architettoniche?

Il contributo affronta il tema della rigenerazione del patrimonio architettonico pubblico attraverso due esperienze emblematiche, tra loro intimamente connesse, riguardanti il Complesso di San Michele a Ripa Grande a Roma, frutto di diverse addizioni e accrescimenti, ma riconducibile a Carlo Fontana, interprete tra i più raffinati della stagione artistica italiana a cavallo tra Sei e Settecento; e il Reale Albergo dei Poveri a Napoli, voluto da Carlo III di Borbone alla metà del XVIII secolo per ospitare, a fini riabilitativi, i poveri del Regno. L'incarico fu affidato a Ferdinando Fuga, reduce dalla realizzazione del Carcere femminile (1734) in San Michele a Ripa Grande, delineandosi un'evidente linea di continuità, non solo tematica, con il precedente edificio romano.

Entrambe le strutture segnano, per mole e qualità del linguaggio adoperato, il tessuto urbano circostante costituendo, in anticipo sui tempi, esempi imponenti di quelle architetture dell'Illuminismo pensate e progettate per assolvere a funzioni pubbliche e sociali.

La loro riconversione, avvenuta attraverso molteplici trasformazioni e usi spesso impropri, ha continuato a interrogare le rispettive città fino a quando il San Michele non è stato destinato a sede degli Uffici del Ministero per i Beni Culturali e Ambientali (1975) e a Centro Internazionale per la Conservazione e il Restauro dei Beni Culturali (I.C.C.R.O.M.) 
-adibendo parte degli spazi all'Istituto Centrale per il Catalogo e la Documentazione, ai laboratori dell'Istituto Centrale del Restauro e a sale per le attività culturali e artistiche-; il caso napoletano, dopo alterne vicende e diversi restauri l'ultimo dei quali ancora in corso, ha individuato nella città dei giovani (2004-2006) l'obiettivo finale del progetto ${ }^{3}$. Intorno alle grandi corti si potranno frequentare corsi di studio universitari, fruire di servizi di assistenza per lo studio e il lavoro, promuovere la collaborazione tra giovani provenienti da diversi paesi, privilegiando spazi quanto più versatili e polivalenti.

Ad accomunare i due edifici concorrono aspetti riguardanti le finalità non solo riabilitative ch'essi furono chiamati a ricoprire nel tempo. L'intento comune di rieducare le fasce più povere della società sottraendole ai pericoli della strada si saldava con la volontà di provvedere all'avvio di processi produttivi capaci d'innescare una crescita economica e culturale delle aree marginali ed emarginate entro le quali tali strutture s'inserivano, modificandone i settori trainanti destinati a trasformarsi rapidamente. Segno caratterizzante di tali Istituti fu proprio il porsi come poli di sviluppo e di coesione sociale rispetto al territorio, creando le condizioni affinché attorno ad essi sorgessero attività artigianali e mestieri in grado di risollevare l'economia urbana in crisi, determinando occasioni di lavoro e di sviluppo per un numero sempre crescente di abitanti.

Il tema dell'assistenza ai poveri non era certamente nuovo alla metà del Seicento e rientrava in quel programma di sensibilizzazione al recupero sociale di intere fasce di bisognosi e abbandonati che, dalla Francia al resto dell'Europa, si sarebbe diffuso rapidamente individuando negli Ospizi e nelle Case d'Internamento le soluzioni adottate per limitare e correggere i danni derivanti dall'accattonaggio e dalla miseria. Proprio il numero di tali Istituzioni può considerarsi un efficace parametro di valutazione per comprendere l'importanza assunta da un centro cittadino rispetto agli altri: quanto più essi erano produttivi ed economicamente indipendenti tanto maggiore era il numero di strutture assistenziali sorte al loro interno per arginare i problemi di sicurezza e di ordine sociale. La consapevolezza della necessità d'individuare nel lavoro una funzione riabilitativa più efficace del mero isolamento spinse alla "condanna etica dell'ozio» (Foucault, 1996: 85). Non si trattava più di «rinchiudere i senza lavoro, ma di dare lavoro a coloro che erano stati rinchiusi, rendendoli partecipi della prosperità comune» (Foucault, 1996: 85). Il significato di tale operazione era più economico che etico e consisteva nel fornire manodopera a basso costo nei periodi di piena operatività, e nel formare e tenere impegnati i reclusi nei periodi di crisi (cfr. Foucault, 1996).

Proprio «la capacità di tali strutture [...] di adattarsi ai nuovi scenari socio-economici in rapida trasformazione segnerà il loro destino: il declino, quando la loro funzione si restringerà ad un amorfo impegno assistenziale, oppure un ruolo attivo, allorché esse riusciranno a integrarsi nei processi industriali in atto già dalla seconda metà del XVIII secolo, e a recuperare fuori dalle mura un più articolato rapporto con la società civile» (Ziviello e D’Arbitrio, 2001: 246).

Lette da questa angolatura, le vicende evolutive del Complesso di San Michele a Ripa Grande presentano aspetti che possono indurci a guardare al caso romano come a un possibile modello di riferimento in tema di reusing di un edificio pubblico restituito nel tempo a una sua rinnovata funzione sociale e formativa, in grado di promuovere attivamente lo sviluppo del territorio urbano circostante. «Un'azienda culturale», in definitiva, che, reinterpretando lo spirito fondativo dell'antica Istituzione, è divenuta il segno tangibile di un intervento di riuso capace di trasformare Trastevere in quartiere direzionale della cultura, riorientando l'asse di sviluppo strategico della capitale.

\section{Complesso del San Michele a Ripa Grande 4}

Articolata in cinque corpi di fabbrica alti quattro piani - internamente organizzati intorno a un «sistema» di corti ${ }^{5}$-, alternati a quattro corpi più bassi posti in corrispondenza delle saldature tra i diversi blocchi, la monumentale facciata del Complesso di San Michele a Ripa Grande segna per trecentocinquanta metri il Lungotevere romano, sottolineando la scala «paesaggistica» dell'intervento. Una dimensione che l'affresco della sala Riaria nel Palazzo della Cancelleria racconta con efficacia restituendo l'immagine di un edificio che, a dispetto della sua storia «frammentata», per compiutezza e omogeneità sembra appartenere al disegno di un unico ideatore [1].

Sorto per volere di monsignor Tommaso Odescalchi alla fine del XVII secolo, il Conservatorio per Ragazzi fu trasferito già dal 1686 in prossimità dell'antico porto fluviale di Ripa Grande, in un'area libera e a basso costo dove poter avviare un più ampio programma di assistenza ai giovani abbandonati. 


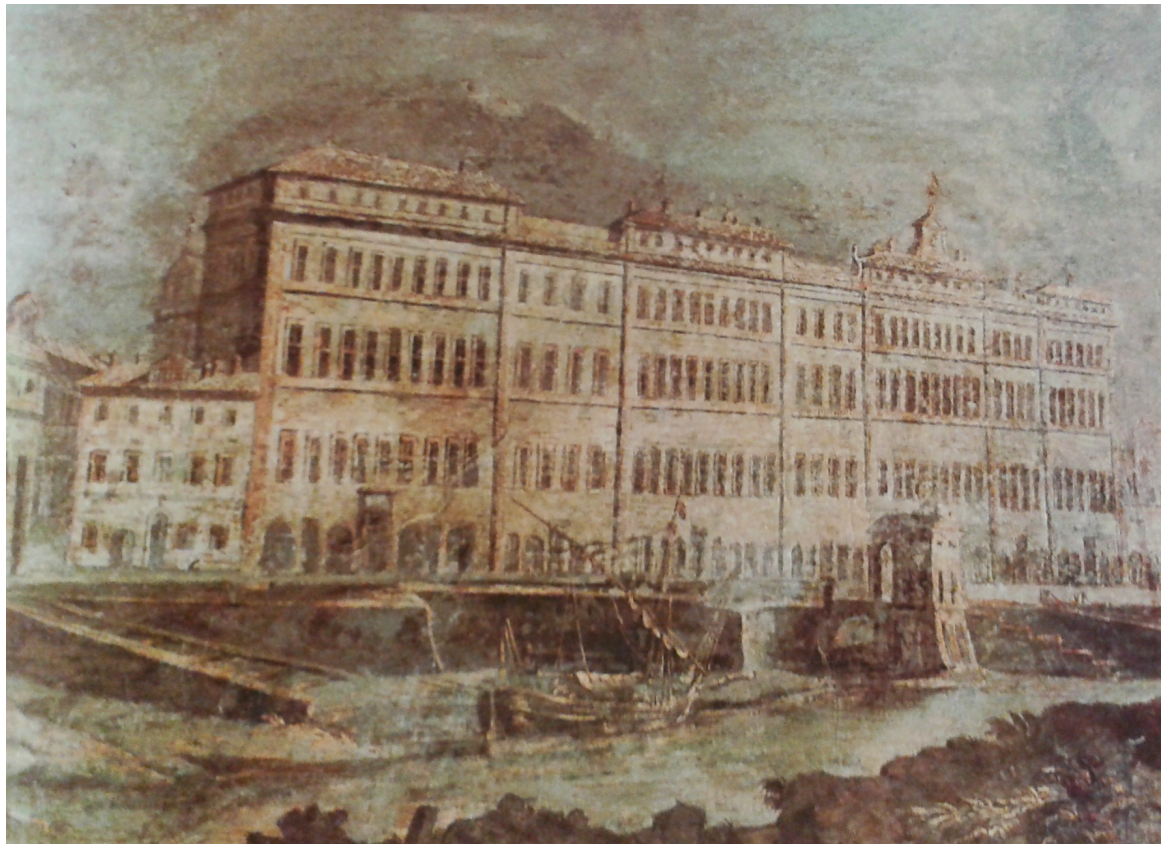

1. Roma, palazzo della Cancelleria, sala Riaria, affresco raffigurante l'Ospizio Apostolico di San Michele sul Lungotevere di Ripa Grande
Inaugurato nella primavera del 1689, con bolla di papa Innocenzo XII del 1693, fu trasformato in Ospizio Apostolico dei Poveri Invalidi con l'intento di accorpare in un unico edificio tutte le organizzazioni pie allora attive in città e di avviare «i poveri orfani [...] nell'opere meccaniche, e lavorieri di lana, leggere, scrivere, far di conti» (Curcio, s.d.: 44). II progetto previde la trasformazione della «sede transtiberina [...] in un moderno opificio, in cui l'educazione e il lavoro dei ragazzi ospiti divenissero funzionali anche all'innovazione della produzione delle «pannine» della città di Roma, al fine di adeguarla a quelle delle maggiori città europee» (Curcio, s.d.: 44).

A tale scopo, il primo nucleo del Conservatorio, coagulato intorno a un cortile quadrato, venne soprelevato e i locali preesistenti riattati. A questo periodo risalgono la ristrutturazione dei locali destinati alla lavorazione e conservazione della lana e la trasformazione della tintoria in ambienti per la lavorazione dei cappelli (Curcio, nota 49: 47). Cosicché il primo nucleo dell'Ospizio andò configurandosi attorno al cortile dei Ragazzi, vero e proprio snodo e centro nevralgico di smistamento e di organizzazione funzionale della fabbrica, su cui immettevano il refettorio, gli opifici e la tintoria.

Moderno luogo di produzione, esso rappresentò sin dal suo nascere un esempio di riconversione in corso d'ope- ra di un edificio sorto per fini assistenziali e tramutato in laboratorio per la formazione e l'avviamento professionale dei giovani reclusi, esempio tangibile di rigore e carità.

La stessa Casa di Correzione Maschile [2], realizzata da Carlo Fontana al suo interno per rieducare i giovani condannati a pene detentive, presentava ambienti adibiti alla filatura e alla tessitura della lana, introdotti per coinvolgere i detenuti nelle attività produttive impiantate nell'edificio, arrivando a impiegare persone «chiamate anche da fuori nei periodi di maggiore attività» (Pietrangeli, s.d.: 181).

Nel 1701 papa Clemente XI Albani incaricava Carlo Fontana di avviare la costruzione del Carcere di Correzione maschile, terminato già nel 1704. L'edificio può essere considerato il prototipo di una concezione moderna di reclusorio per la quale il detenuto non era destinato unicamente a scontare la condanna inflittagli, ma se ne prevedeva il reinserimento sociale quale soggetto recuperabile chiamato a integrarsi pienamente nella collettività.

L'organizzazione spaziale del carcere era stata concepita per assolvere tanto alle funzioni correttive - con la disposizione laterale delle celle servite da due ordini di ballatoi sovrapposti, secondo un modello ispirato alla tipologia degli edifici conventuali -, quanto alle funzioni produttive fi- 
nalizzate alla filatura della lana, assolte dalla spina centrale della sala-corridoio rettangolare a tutta altezza, illuminata dall'alto su cui affacciavano, secondo una «disposizione a palchi» (Amirante, 2001: 204), i ballatoi di accesso alle celle da cui era possibile assistere alle funzioni religiose celebrate nella cappella posta a conclusione del rettangolo. La risonanza di tale intervento è testimoniata dalla tavola che ne raffigura la pianta e sezione pubblicata da John Howard nel suo fortunatissimo volume Etat des prisons, des hopitaux, des maisons de force, edito a Parigi nel 1788 e destinato a divenire rapidamente uno dei testi di riferimento in tema di moderne strutture per la riabilitazione e l'inclusione sociale degli assistiti.

Alla metà del XIX secolo all'interno dell'Ospizio di San Michele trovavano luogo, accanto alle strutture assistenziali, il lanificio per la produzione del lino e dei tessuti adoperati per le divise dei corpi papali; la lavorazione della seta e la fabbrica degli arazzi destinati ad adornare i palazzi apostolici; la stamperia, che deteneva il monopolio dei libri di testo delle scuole pontificie; la legatoria; le botteghe d'arte. L'apprendimento delle arti meccaniche si accompagnava a quello delle arti liberali il cui insegnamento era affidato a docenti selezionati tra gli Accademici di San Luca. La creazione di

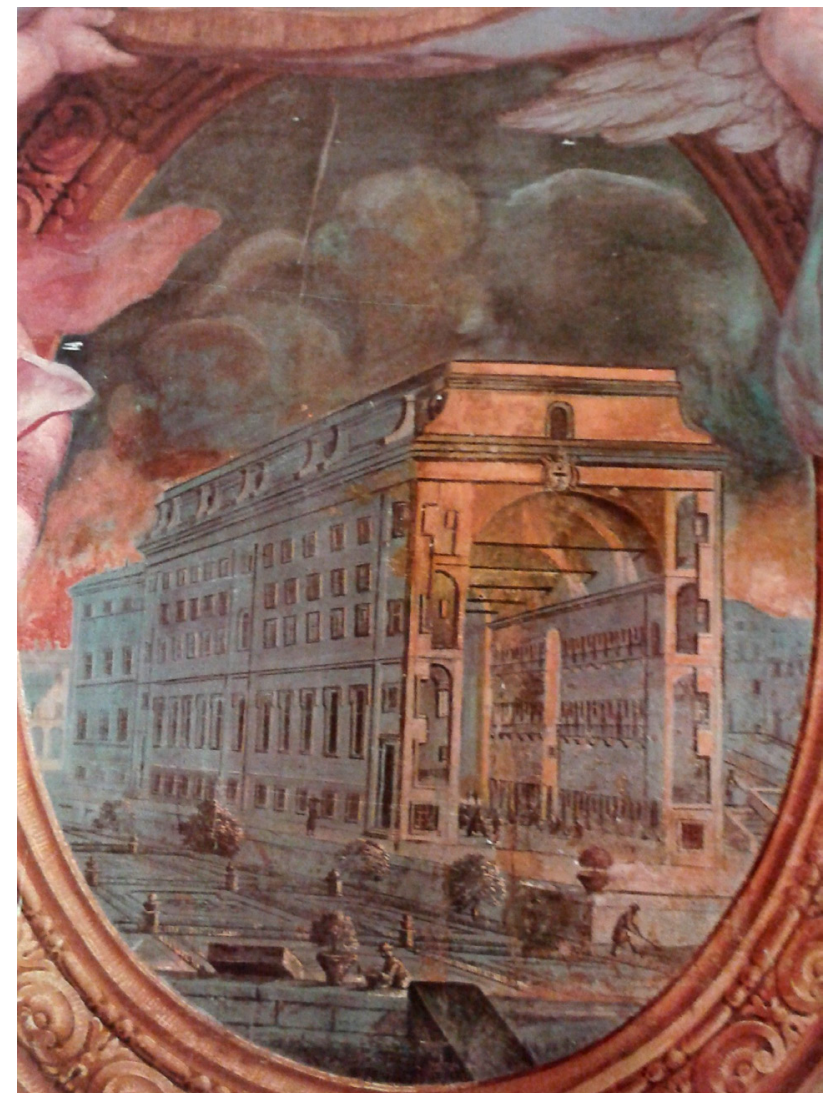

2. Roma, palazzo della Cancelleria, sala Riaria, affresco raffigurante la sezione-prospetto dell'Ospizio di San Michele a Ripa con in sezione il Carcere minorile progettato da Carlo Fontana
3. Giuseppe Vasi, II Porto di Ripa Grande, tratto dalle

Magnificenze di Roma Antica e Moderna, Libro V, Roma 1747-1761

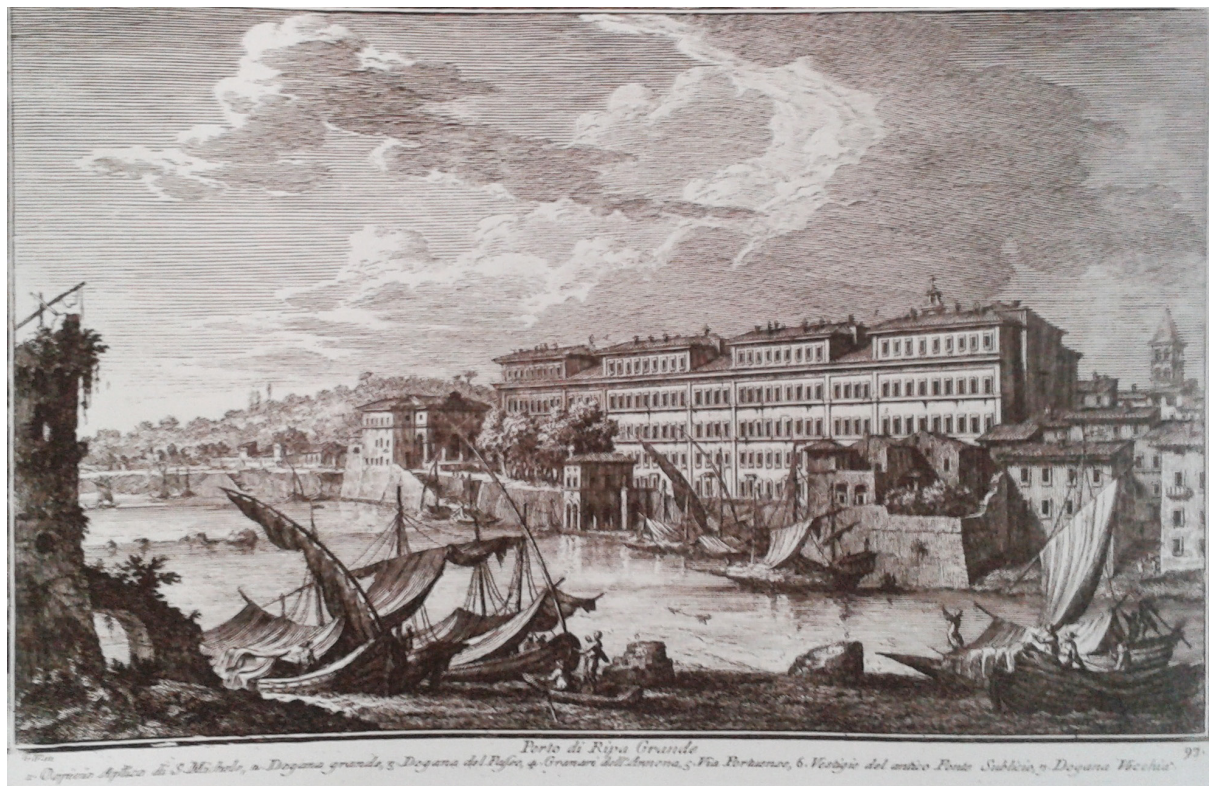




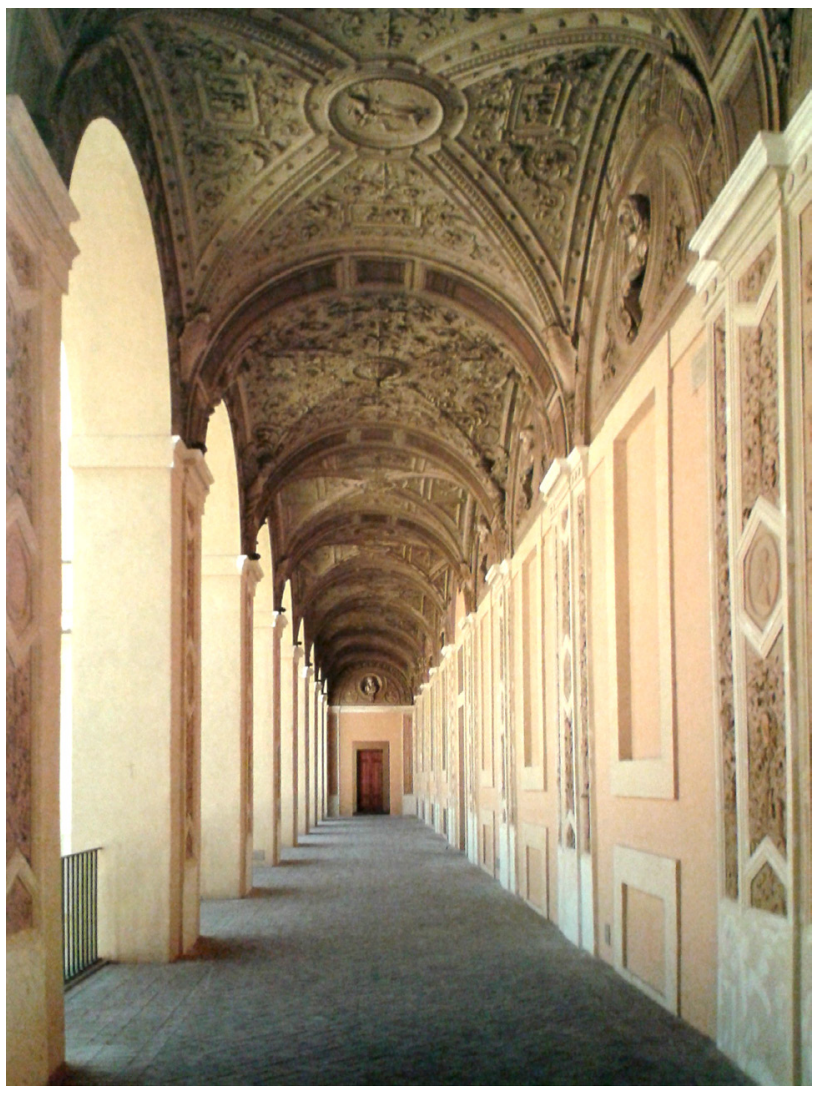

4. Roma, Complesso di San Michele a Ripa, il Loggiato degli Stucchi nel cortile del Conservatorio dei Vecchi dopo gli interventi di restauro

scuole e laboratori di scultura, di disegno dal vero, di figura e di ornato; la collezione di copie dall'antico; l'apprendimento dell'arte delle medaglie, dell'intaglio di marmi, di pietre e di legno; la fonderia, contribuirono alla formazione di un ceto artigianale altamente specializzato e professionalizzato, indispensabile per la crescita economica della città. Già nel XVIII secolo «Roma [...] possedeva il più grande centro di assistenza sociale di tutto lo Stato Pontificio, [...] che con il passare del tempo divenne anche uno dei più grandi opifici della capitale» (Toscano, 1996: 162).

Quando negli anni Settanta del Novecento l'edificio fu adibito a Ministero per i Beni Culturali e Ambientali ospitando, negli ambienti disposti tra il cortile dei Ragazzi e i cortili delle Arti e dei Marmi storicamente adibiti ai laboratori-scuole, le aule dell'Istituto Centrale del Restauro, l'intima coerenza tra le finalità iniziali del Complesso del San Michele e il suo riu- so determinò la riuscita del progetto di ri-funzionalizzazione dell'antico Ospizio Apostolico convertito al ruolo «laico» di moderna officina di saperi e di mestieri, modello di riferimento per futuri interventi di tutela e valorizzazione del patrimonio storico-artistico proiettati su scala urbana.

La ristrutturazione funzionale del Complesso di San Michele costituisce, infatti, un esempio rigoroso del modo di relazionarsi, in sede di progetto, alle "caratteristiche architettoniche, spaziali e strutturali degli ambienti» (De Tomasso e Marchetti, 1983: 102-141) oggetto di tutela, la cui conformazione e tipologia originaria era composta di corpi di fabbrica di epoca diversa coagulati intorno a cortili di smistamento [3].

Consolidamento strutturale, restauro e rifunzionalizzazione graduale dell'edificio hanno costituito tre fasi inscindibili di un unico progetto di recupero tendente a rispettare le relazioni di continuità intercorrenti tra la disposizione degli ambienti e le loro «vocazioni formative».

Attualmente gli ambienti destinati ad accogliere gli uffici e le strutture della Soprintendenza sono disposti intorno al cortile dei Ragazzi e dei Vecchi [4]; I'Istituto Centrale per il Restauro - composto di spazi destinati alla ricerca scientifica, alla conservazione e al restauro dei beni architettonici e storico artistici - è sistemato tra il cortile dei Carceri, il cortile delle Arti e dei Marmi e il cortile dei Ragazzi, attorno ai quali gravitano anche le aule e gli ambienti per lo svolgimento dei corsi di insegnamento, con i laboratori di pittura posti nel blocco dei ragazzi sul Lungotevere. L'Istituto Centrale per il Catalogo e la Documentazione occupa gli spazi intorno al cortile delle Zitelle; mentre I'ICCROM, organo internazionale di ricerca, formazione e archiviazione dati, gravita intorno al cortile del Porto, con la sala conferenze disposta negli ambienti un tempo destinati a Stenditoio. La chiesa incompiuta di Carlo Fontana, fulcro e cuore dell'Ospizio, svolge le funzioni di ambiente polivalente destinato alle manifestazioni culturali e scientifiche, mentre i corpi delle Case di Correzione Maschile e Femminile sono stati adibiti a funzioni museali per preservarli da possibili alterazioni.

La funzionalità del Complesso di San Michele, organizzato secondo precisi criteri distributivi, ha consentito di recuperarne «'organigramma funzionale», garantendo il contemporaneo recupero della sua memoria storica e il ruolo di struttura per il sostegno, la promozione e lo sviluppo di attività e servizi per la collettività. 


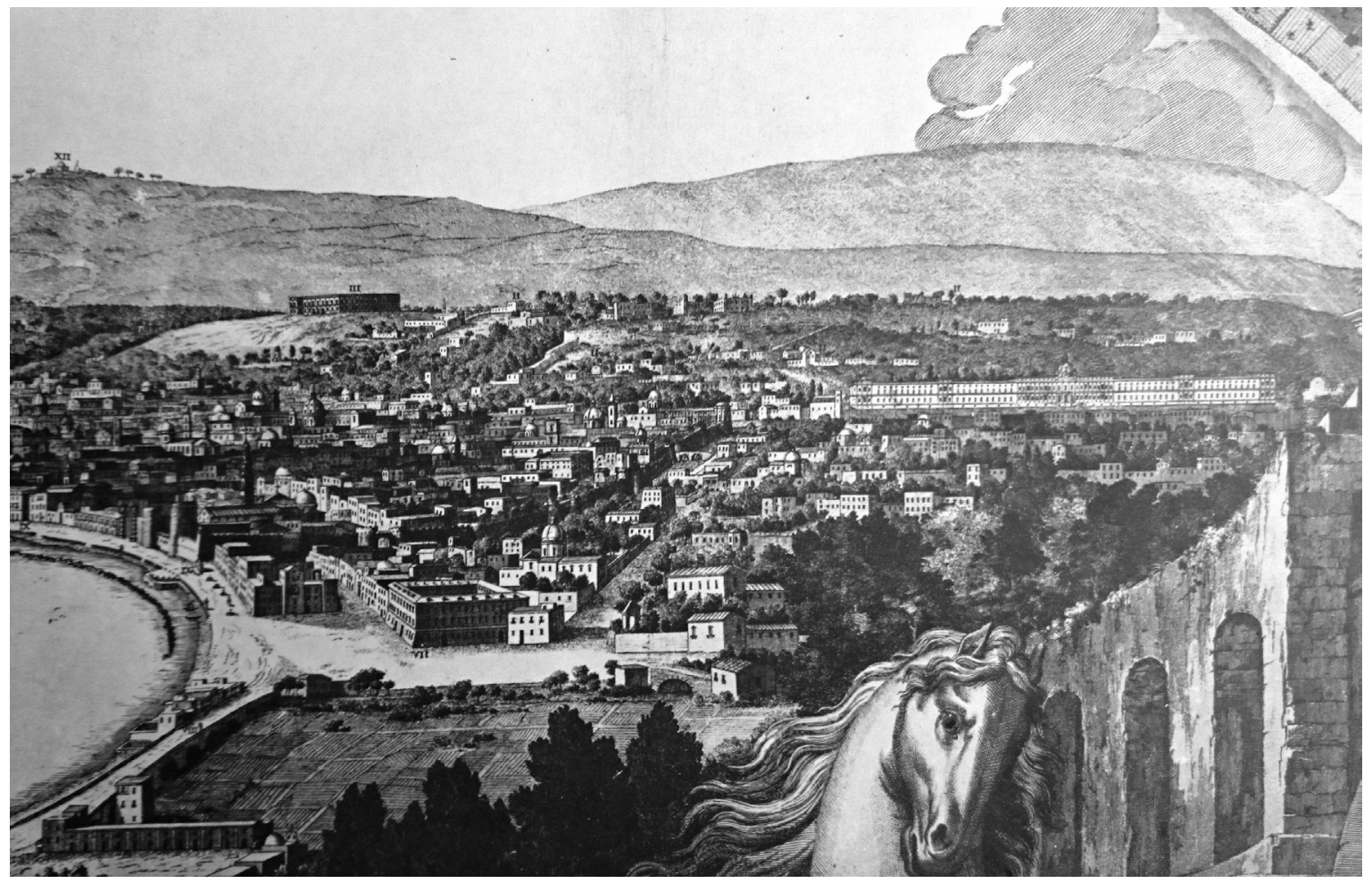

5. Veduta scenografica a ponente della città di Napoli in Campagna Felice, particolare della pianta del Duca di Noja, Napoli 1775

\section{Reale Albergo dei Poveri a Napoli}

Fu per assolvere a problemi di tenuta sociale che nel 1749 Carlo III di Borbone incaricò Ferdinando Fuga - reduce dai cantieri romani del San Michele a Ripa Grande e dall'ampliamento dell'Ospedale di Santo Spirito in Sassia (1742-44) - di costruire a Napoli il più grande Ospizio d'Europa, capace di accogliere ottomila emarginati, fornendo loro condizioni di vita e di lavoro adeguate. «Fu destinato per ottomila Poveri, da ripartirsi in quattro ceti, cioè di uomini, di donne, di ragazzi, e di ragazze, senza alcuna communicazione fra loro. Annessa al suddetto Ospizio egli architettò una vasta Chiesa pubblica, da frequentarsi divisamente dai quattro ceti suddetti. Grandi vi sono i comodi per lavoratorj, refettorj, cortili, portici, officine, e abitazioni per i Serventi e per i Ministri addetti» (Milizia, 1785, II, 290).

L'idea di costruire un edificio-città, concepito come un organismo comunitario autosufficiente, si riverberò sulla natura stessa dei progetti proposti. Sia che si guardi il primo pro- getto, basato su di un impianto quadrato suddiviso internamente in quattro corti, da erigere nel borgo di Loreto, nell'area orientale di Napoli; sia che si osservi il progetto definitivo, organizzato sulla successione di cinque maestosi cortili-piazze, da edificare nel borgo di Sant'Antonio Abate, in un'area cruciale per i collegamenti con Roma e i traffici cittadini, non si può non considerare l'importanza che la nuova fabbrica era chiamata a ricoprire negli intenti del sovrano. Ubicato ai piedi della collina di Capodimonte lungo il prolungamento del viale che conduceva alla sontuosa villa di Poggioreale [5] $]^{6}$, una tra le abitazioni più eleganti e celebrate del primo rinascimento napoletano, l'edificio, di cui vennero realizzati soltanto i tre cortili centrali, gareggiava per dimensioni (seicento metri per centoquaranta) e linguaggio architettonico con la residenza reale di Caserta, ultimo esempio "anacronistico» di cittàcapitale dell'assolutismo monarchico [6] .

Le vicende costruttive dell'Albergo dei Poveri si snodano lungo un arco temporale che va dalla morte di Ferdinando Fuga (1782), a Mario Gioffredo, all'intervento di Car- 


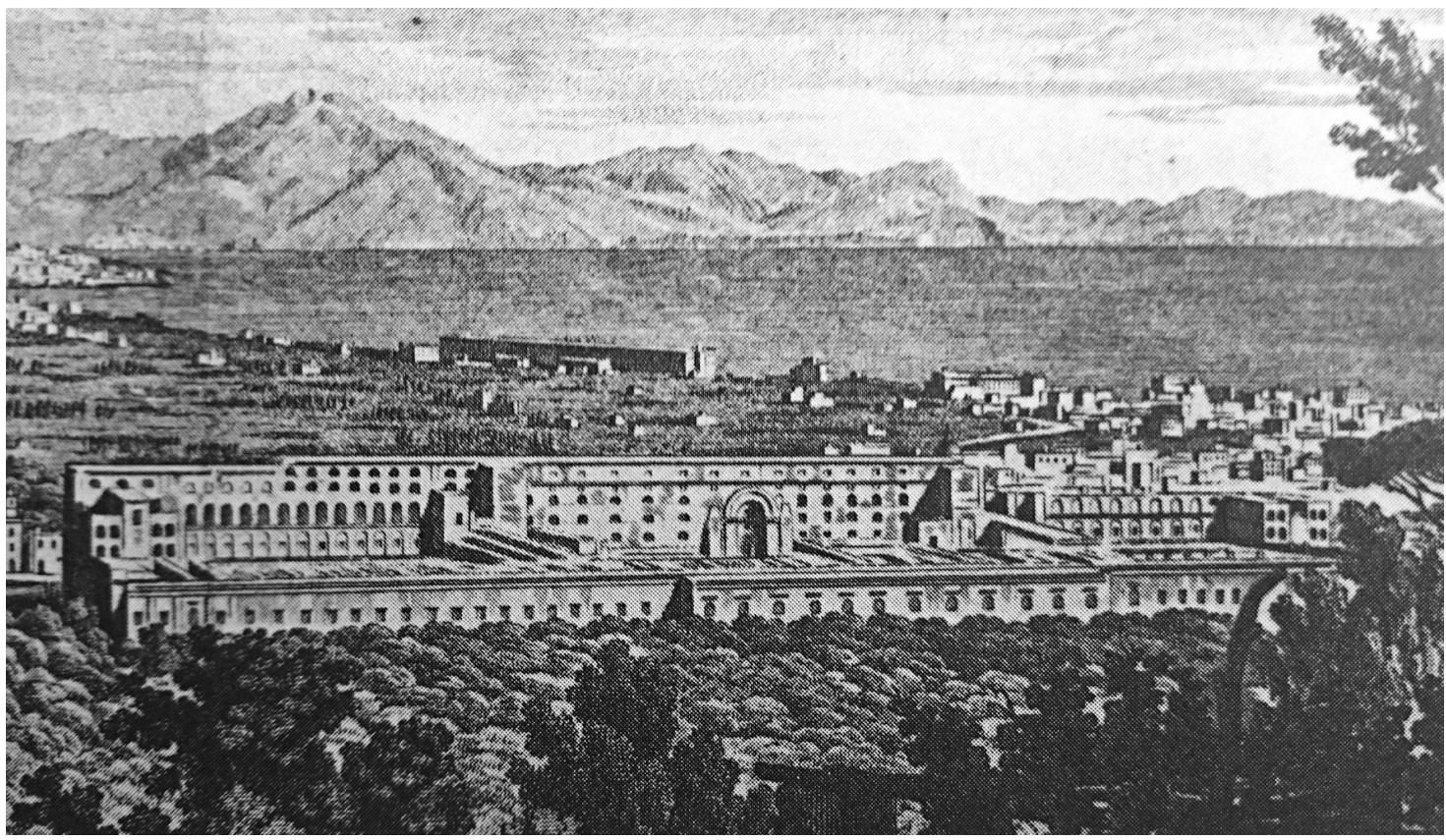

6. Le due grandi fabbriche edificate da Ferdinando Fuga a Napoli nella seconda metà del XVIII secolo

lo Vanvitelli (1785-1799) e da questi a Francesco Maresca (1802), autore del definitivo ridimensionamento del progetto fughiano.

II linguaggio architettonico adottato dall'architetto fiorentino suggerisce una doppia chiave interpretativa: da un lato il filone tardobarocco, rinvenibile nella incompiuta chiesa a Panoptikon posta al centro del cortile intermedio; da un altro il filone classicista con cui è risolto lo sviluppo dell'intero edificio, come i Granili (cinquecentocinquanta metri) giocato sulla poetica della grande dimensione e sulla scala paesaggistica dell'intervento. La pianta della chiesa nella prima soluzione prevista per il borgo di Loreto presentava una serie di ballatoi interni aperti sulle navate secondo un'organizzazione sperimentata da Fontana proprio nella chiesa di San Michele a Ripa, di cui veniva ripresa anche la suddivisione per classi degli spazi interni. Nel passaggio al sito definitivo, anche il progetto della chiesa si complica considerevolmente con la posizione della cupola che subisce un arretramento rispetto alla più svettante e incombente soluzione iniziale.

Le dimensioni dell'edificio, così come parzialmente realizzato, presentano un'ampiezza di facciata pari a trecen- tosessanta metri di lunghezza per centoquaranta metri di larghezza; con una superficie di centotremila metri quadrati e un volume di ottocentotrentamila metri cubi.

Con i suoi quattrocentotrenta ambienti e ventimila metri quadrati di spazi all'aperto ${ }^{8}$, la struttura, dalla mole colossale, ben più imponente della fabbrica romana, è da sempre considerata un "passo falso» nelle politiche governative dei Borbone. «Chi sa quando si finirà? E sono quasi trent'anni, che si lavora a quest'opera. Con minore spesa, e in più breve tempo si sarebbe tolta per sempre ogni povertà all'abbondantissimo Regno di Napoli. Ma questo non è affare dell'Architetto, ma del buon Governo» (Milizia, II: 290).

Eppure, visto dalla tangenziale, l'Albergo dei Poveri trasmette nitido il senso della sua costruzione. La proporzione ch'esso instaura con la città metropoli che le è sorta intorno racconta con efficacia ineguagliabile il significato dell'operazione strategica condotta a suo tempo dai Borbone: era una città nella città che s'intendeva costruire, la «città dei servizi», posta all'ingresso della capitale del regno [7]. Più che gli aspetti costruttivi dell'Albergo dei Poveri, interessa qui sottolineare il ruolo propulsore ch'esso fu chiamato a ricoprire rispetto al territorio circostante quando, sull'onda 
della rivoluzione industriale e dei nuovi modi di produzione, divenne «incubatore» d'imprese e di mestieri.

Già Ferdinando IV di Borbone aveva creato le premesse affinché al suo interno si avviasse un processo di industrializzazione teso a individuare nei due poli della formazione professionale e dell'istruzione i punti cardine attorno a quali far ruotare, a fini produttivi, il riutilizzo funzionale dell'edificio. L'avvento dei francesi e il ritorno dei Borbone segnarono gli anni di maggiore produttività e «flessibilità d'uso" dell'Albergo dei Poveri. L'edificio divenne il centro di una strategia finalizzata «a costituire un efficace luogo di intersezione tra funzione programmatica di pubblico interesse e [...] ruolo esecutivo dei privati» (Ziviello e D’Arbitrio, 2001: 254). Non soltanto fu sede di diverse attività produttive ma divenne polo produttivo collegato ad altre importanti istituzioni dislocate sul territorio grazie a una serie di contratti e attività che ne regolamentavano i rapporti: una sorta di clusters che lavoravano in sinergia con la sede centrale. È il caso della fabbrica di coralli, avviata a Torre del Greco ma impiantata con una propria officina indipendente anche nell'Albergo dei Poveri, o della scuola di nautica che, avviata dal marchese Tanucci, vide nel 1816 la sua fioritura grazie proprio alla collaborazione con i giovani dell'Albergo.

D'altra parte fu proprio durante il periodo borbonico che si andò affermando l'idea d'impiantare nel regno di Napoli una «filiera culturale diffusa» ${ }^{9}$. Dall'editoria, ai sistemi produttivi locali, dalle seterie ai laboratori nell'Albergo dei Poveri, si delinea la messa a punto di un formidabile sistema produttivo distribuito sul territorio che individuava nelle radici culturali della tradizione storico-artistica e artigianale locale le leve per lo sviluppo e la crescita futuri. Le seterie di San Luecio, l'azienda agricola di Carditello, le fabbriche di coralli e di ceramiche, l'arazzeria, gli opifici per la produzione e la filatura della lana, la stamperia, i laboratori di metalli costituiscono un altro modo per leggere in filigrana la storia della cultura architettonica partenopea a cavallo del XIX secolo, all'interno della quale un ruolo fondamentale svolse proprio l'Albergo dei Poveri, vera fucina di arti e di mestieri, polo produttivo conficcato nel cuore antico della città.

Il tema, oggi attualissimo, dell'industria culturale quale elemento in grado di immettere, nel più ampio sistema economico, un mercato della bellezza e della cultura fu avviato già dai Borbone non solo quale instrumentum regni, ma come progetto in grado d'innescare processi produttivi ca-

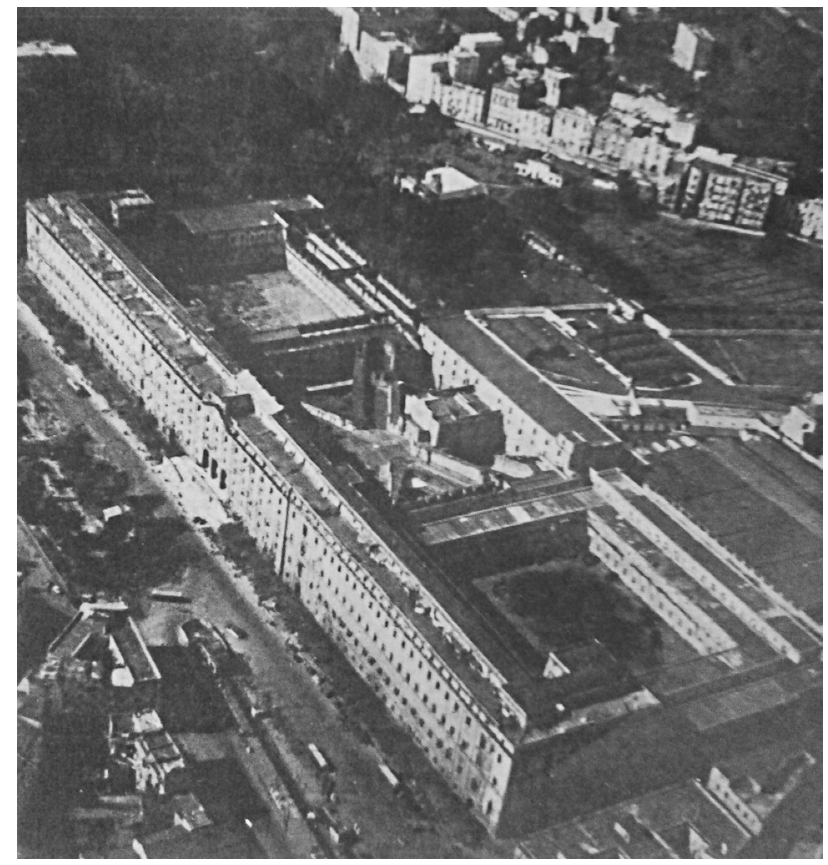

7. Napoli, veduta aerea dell'Albergo dei Poveri. (tratta da R. Pane, Ferdinando Fuga, 1956)

paci di alimentare innovazione, ricerca e creatività all'interno di un «polo della conoscenza» sostenuto dalle politiche del regno. La riscoperta di Pompei e di Ercolano e la campagna di scavi archeologici che ne derivò, con il conseguente rilievo, disegno e pubblicazione delle Antichità di Ercolano esposte, diede il via a studi e ricerche nei più diversi settori della conoscenza e della produzione artistica, donando nuova linfa e ispirazione a pittori, scultori, accademie e allo stesso settore tessile la cui riproduzione di tessuti, stampe e motivi decorativi s'ispirò a quel formidabile repertorio di forme e tradizioni culturali riemerse dagli scavi, dando impulso all'economia e alla produzione artigianale.

Denominato Grande Emporio di Arti e Manifatture svariatissime, l'Albergo dei Poveri svolse un ruolo importante nello sviluppo economico e commerciale della capitale. Alla metà dell'Ottocento «brulicava di attività: [...] dai grandi [...] spazi destinati alle rimesse per il treno della Reale artiglieria, agli spazi per [...] la fonderia, l'incisione, [...] la vetreria, [...] la tintoria delle lane, [...] la sala delle fusioni, [...], la scuola di Belle Arti» (Ziviello e D’Arbitrio, 1999: 206), mostrando la sua 

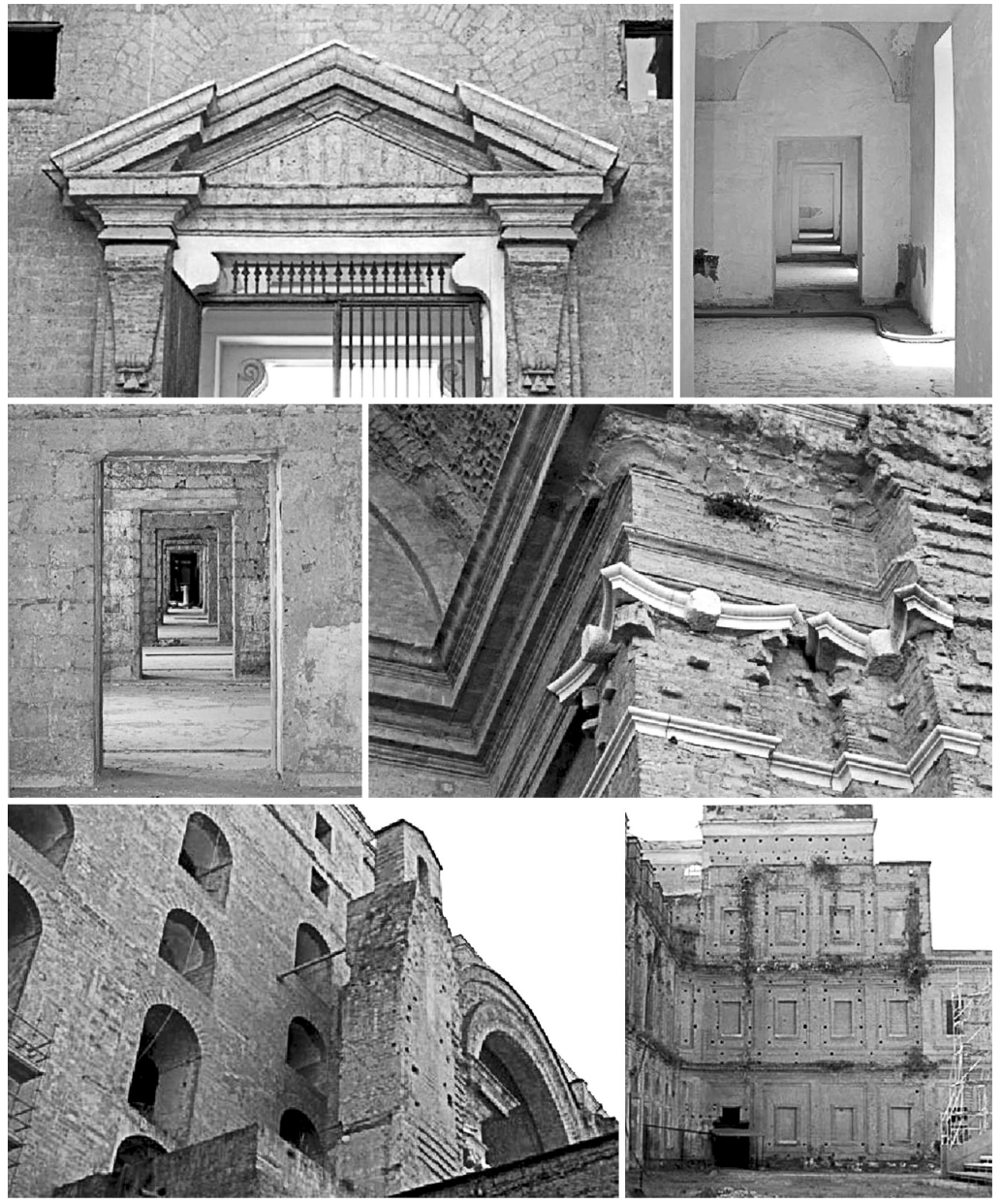

8. Napoli, il Reale Albergo dei Poveri, vedute interne. Dal basso, particolare dell'arcata d'ingresso alla incompiuta chiesa; arcate dei cortili e modanature delle finestre; la fuga delle sale interne prima e dopo gli interventi di restauro natura polifunzionale, conseguenza della struttura modulare dell'edificio.

La razionalizzazione dei percorsi interni, composti di nove chilometri di corridoi; la chiara distribuzione degli ambienti; l'articolazione dei corpi di fabbrica attorno alle tre grandi corti; la ripetizione «seriale» dei moduli di facciata, consentono una ristrutturazione funzionale dell'edificio che ne rispetti le caratteristiche costruttive, mantenendo inalterata la tipologia [8].

Reclusorio, laboratorio, fabbrica, scuola, l'Albergo dei Poveri può garantire un uso flessibile e plurale dei suoi spazi interni, coniugando alcuni dei grandi temi attorno ai quali ruota il riuso degli edifici pubblici da adibire a spazi per la collettività e tenendo conto di una nuova, «antica» emergenza sociale che le cronache dei nostri giorni drammaticamente raccontano: quella dell'accoglienza e della inclusività.

\section{Conclusioni}

II recupero del Complesso romano di San Michele a Ripa Grande, avvenuto per tappe e nel rispetto dei caratteri tipologici dell'edificio, ha consentito di preservarne la memoria storica e il ruolo di struttura per il sostegno, la promozione e lo sviluppo di attività e servizi per la collettività. Analogamente, le politiche di rigenerazione urbana e di riconversione funzionale che interessano da tempo il Reale Albergo dei Poveri a Napoli e che non hanno ancora trovato una loro conclusione dovranno privilegiare un uso flessibile e plurale degli spazi interni, incentivando creatività e contaminazioni culturali in una rinnovata visione pedagogica di un "edificio-città" pensato e progettato come laboratorio permanente di idee, arti e mestieri e destinato, per sua natura e "vocazione», a usi e riusi formativi. 


\section{Notes}

1 L'articolo approfondisce il contributo dal titolo Rigenerare la «funzione sociale». II riuso del Complesso di San Michele a Ripa Grande a Roma e del Reale Albergo dei Poveri a Napoli presentato in occasione del convegno AID Monuments 2015-2 ${ }^{\text {nd }}$ International Conference on Architecture and Engineering for the Defence of the Monuments. Materials Techniques Restoration for Architectural Heritage Reusing (Perugia, 14-16 Maggio 2015).

2 BACCHELLA, Ugo, BOLLO, Alessandro, MILELLA, Franco (2015), «Riuso e trasformazioni degli spazi a vocazione culturale e creativa: un driver per lo sviluppo, ma a quali condizioni?», // Giornale delle Fondazioni, 15 luglio, p. 24, consultabile su www.ilgiornaledellefondazioni.com. Cfr. MANZO Rosario (2005), "ll processo di rivitalizzazione del patrimonio pubblico», in MANZO Rosario, TAMBURINI Gualtiero (a cura di) (2012), II patrimonio immobiliare pubblico. Nuovi orizzonti. II ruolo dell'Agenzia del Demanio, II Sole24Ore, Milano; «Rigenerare le città attraverso la valorizzazione del patrimonio pubblico: tecnologie ambientali e creatività» Technè, vol. 3, Firenze University Press, Firenze, pp. 303-305.

3 Cfr. Master Plan per la realizzazione della «città dei giovani», Comune di Napoli, dicembre 2004, consultabile alla pagina www.comune.napoli.it.

4 II Complesso di San Michele presenta una facciata di larghezza pari a $350 \mathrm{~m}$, con una superficie coperta di 64.400 mq., un volume utile di 343 mc. e tempi di costruzione pari a circa 150 anni, considerando le aggiunte ottocentesche introdotte dal Poletti.

5 Essi possono riassumersi in: il cortile dei Ragazzi; il cortile delle Arti che, assieme al cortile dei Marmi, ai cortili del Carcere Maschile e del Carcere Femminile e al cortile della Dogana, compongono il nucleo meridionale del Complesso; il cortile dei Vecchi, con alle spalle il cortile delle Carrette; il cortile delle Zitelle e il cortile del Porto. Cfr. San Michele a Ripa Storia e Restauro, 1983.

6 L'immagine ritrae una veduta d'assieme della città con in evidenza la mole dell'Albergo dei Poveri raffigurata secondo lo sviluppo previsto nel progetto originario articolato intorno a cinque cortili.

7 In primo piano emerge il volume dell'Albergo dei Poveri come effettivamente realizzato con soli tre cortili centrali e con in evidenza l'arcata d'ingresso dell'incompiuta chiesa; alle spalle si riconosce il blocco compatto della fabbrica dei Granili.

8 Per una approfondita analisi dell'Albergo dei Poveri si rimanda al fondamentale volume di Paolo GIORDANO (2014), L'Albergo dei Poveri a Napoli: il ridisegno, il rilievo e la riconfigurazione dell'architettura monumentale, La scuola di Pitagora, Napoli.

9 Cfr. SERRAGLIO, Riccardo (2001), Sperimentazione e cultura tecnica per i Borbone di Napoli, E.S.I., Napoli.

\section{Bibliografia}

AMIRANTE, Giosi (2001), «La figura e l'opera di Ferdinando Fuga», in GAMBARDELLA A. (a cura di), Ferdinando Fuga 1699-1999 Roma Napoli Palermo, E.S.I., Napoli, pp. 243-255.

CATERINA, Gabriella e DE JOANNA, Paola (a cura di) (2007), /l Real Albergo de' Poveri di Napoli. La conoscenza del costruito per una strategia di riuso, Liguori, Napoli.

CURCIO, Giovanna (s.d.), «Ripa Grande, Frammento di una città nuova», /l San Michele a Ripa Grande, Editalia, Roma, pp. 37-60.

DE TOMASSO, Francesco e MARCHETTI, Patrizia (1983), «ll recupero funzionale», San Michele a Ripa, Storia e restauro, Istituto della Enciclopedia Italiana, Roma, pp. 102-141.

FOUCAULT, Michael (1996), Storia della Follia in età classica, BUR, Milano.

GIORDANO, Paolo (2014), L'Albergo dei Poveri a Napoli: il ridisegno, il rilievo e la riconfigurazione dell'architettura monumentale, La Scuola di Pitagora, Napoli.

MARCHETTI, Patrizia (1981), «ll progetto di restauro», Tre interventi di restauro. San Michele - Convento di San Francesco a Ripa - Santa Cecilia, De Luca, Roma, pp. 24-36.

MARCONI, Paolo (1999), Materia e significato, Laterza, Roma-Bari.

MILIZIA, Francesco (17854), Memorie degli architetti antichi e moderni, 2 voll., Bassano, II.

PIETRANGELI, Carlo (1983), «L'Ospizio apostolico di San Michele nella sua funzione religiosa e sociale», San Michele a Ripa, Storia e restauro, Roma, pp.175-184.

S.A. (2004), «Master Plan per la realizzazione della 'città dei giovani'», Comune di Napoli, dicembre, http://www.comune.napoli.it.

SERRAGLIO, Riccardo (2001), Sperimentazione e cultura tecnica per i Borbone di Napoli, E.S.I., Napoli.

TAMIOZZO, Raffaele (2002), Restauro e conservazione. L'enciclopedia Treccani on line, http:// www.treccani.it.

TOSCANO, Pia (1996), Roma produttiva tra Settecento e Ottocento // San Michele a Ripa, Viella, Roma.

VIOLLET-LE-DUC, Eugène Emmanuel (1982), L'architettura ragionata. Estratti dal dizionario, Jaca Book, Milano.

W.AA. (s.d.), I/ San Michele a Ripa Grande, Istituto Poligrafico e Zecca dello Stato, Editalia, Roma.

ZIVIELLO, Luigi e D'ARBITRIO, Nicoletta (1999), Il Reale Albergo dei Poveri di Napoli: un edificio per le arti della città. Dentro le Mura, Edisa, Napoli.

- «ll Reale Albergo dei Poveri di Napoli: un edificio per le arti della città», Ferdinando Fuga 1699-1999 Roma Napoli Palermo, E.S.I., Napoli, pp. 243-255. 
